\title{
A gap method for increasing the sensitivity of cantilever biosensors
}

Stephane Leahy and, and Yongjun Lai

Citation: Journal of Applied Physics 122, 064502 (2017); doi: 10.1063/1.4989959

View online: http://dx.doi.org/10.1063/1.4989959

View Table of Contents: http://aip.scitation.org/toc/jap/122/6

Published by the American Institute of Physics

\section{AlP | Journal of A1P Aplied Physics}

Save your money for your research.

It's now FREE to publish with us no page, color or publication charges apply.
Publish your research in the

Journal of Applied Physics

to claim your place in applied

physics history. 


\title{
A gap method for increasing the sensitivity of cantilever biosensors
}

\author{
Stephane Leahy and Yongjun Lai ${ }^{\mathrm{a})}$ \\ Department of Mechanical and Materials Engineering, Queen's University, Kingston, Ontario K7L3N6, \\ Canada
}

(Received 13 June 2017; accepted 30 July 2017; published online 11 August 2017)

\begin{abstract}
Dynamic-mode cantilever biosensors are an attractive technology for biological sensing. However, researchers are constantly seeking ways to increase their sensitivity, especially in liquids, in order to rapidly detect biological particles in dilute samples. Here, we investigate a novel gap method for increasing their sensitivity. We design cantilevers with a micron-sized gap between their free end and a supporting structure that constrains the motion of individual particles deposited across the gap. When individual particles are deposited across the gap, they form a bridge between the free end of the cantilever and the supporting structure and generate fundamental resonant frequency shifts that are two orders of magnitude higher than when they are deposited on conventional cantilevers. Through experiments and simulation in air, we show that cantilevers based on the gap method rely on stiffness-change instead of mass-change, and that they are significantly more sensitive than conventional cantilevers. Published by AIP Publishing. [http://dx.doi.org/10.1063/1.4989959]
\end{abstract}

\section{INTRODUCTION}

Dynamic-mode cantilever biosensors are an attractive technology for biological sensing because they are labelfree, highly sensitive, and can be mass-produced at low cost. $^{1-5}$ With applications looking to measure smaller and smaller particles and to make measurements at lower and lower concentrations, researchers are constantly seeking ways to improve the sensitivity of dynamic-mode cantilevers. The physics describing these devices is well understood; it shows that when their mass increases, their resonant frequency decreases, and that their sensitivity $(\mathrm{Hz} / \mathrm{g})$ is related to their resonant frequency, mode order, and quality factor. $^{6}$

For measurements in air or a vacuum, significant improvements in sensitivity have been achieved by miniaturizing cantilevers down to the nanoscale. Using such scales, single bacteria ${ }^{7}$ and viruses ${ }^{8}$ have been detected, and attogram $^{9}$ and zeptogram ${ }^{10}$ sensitivities have been demonstrated. For measurements in liquids, however, miniaturization has led to much more modest improvements in sensitivity due to the dominating effect of viscous damping at smaller scales. ${ }^{11}$ Therefore, other techniques have been developed to increase sensitivity. These include using a resonant frequency tracking circuit $^{12,13}$ using the higher order resonant modes of a millimeter-sized cantilever, ${ }^{14-20}$ or integrating a suspended microchannel into a cantilever. ${ }^{21,22}$ These techniques have provided sensitivities high enough for measuring a single bacterium. However, they can be difficult to implement, are not necessarily adapted to standard micromachining processes, and still leave much to be desired in terms of sensitivity.

Here, we study a new technique called the gap method for increasing the sensitivity of cantilever biosensors. We designed cantilevers that have a micron-sized gap between their free end and a supporting structure. The supporting structure, which we call the particle anchor, serves to

a)1ai@queensu.ca constrain the motion of individual particles that are deposited across the micron-sized gap. When individual particles are deposited across the gap, they greatly increase the resonant frequency of the cantilever. Experiments and simulations suggest that cantilevers based on the gap method rely on stiffness-change instead of mass-change, and are significantly more sensitive than conventional cantilevers.

\section{METHODS}

\section{Theory}

The gap method is based on the following model of a cantilever [see Fig. 1(a)]. We assume that the cantilever is made of an isotropic material, and that it has a large aspect ratio, a small deflection compared to its thickness, and a single-layer uniform rectangular cross-section. We also assume that damping is negligible. ${ }^{23}$

Under these assumptions, the bending resonant frequencies of the cantilever are

$$
f_{n}=\frac{t \lambda_{n}^{2}}{4 \pi L^{2}} \sqrt{\frac{\mathrm{E}}{3 \rho}}=\frac{1}{2 \pi} \sqrt{\frac{k_{\mathrm{eff} n}}{m_{\mathrm{eff} n}}}
$$

where $n$ is the bending mode order, $t$ is the thickness, $\lambda_{n}$ is the solution to $1+\cos \left(\lambda_{n}\right) \cosh \left(\lambda_{n}\right)=0, L$ is the length, $\mathrm{E}$ is the Young's modulus, $\rho$ is the density, $k_{\mathrm{eff} n}$ is the effective stiffness and $m_{\operatorname{eff} n}$ is the effective mass. The right-hand solution is the resonant frequency of the cantilever expressed in the form of an equivalent harmonic oscillator model. The harmonic oscillator model is useful for calculating the fundamental resonant frequency shift caused by the deposition of a particle onto the free end of the cantilever. For the fundamental resonant frequency, the effective stiffness of the cantilever is

$$
k_{\mathrm{eff} 1}=\frac{E w t^{3}}{12 L^{3}}
$$




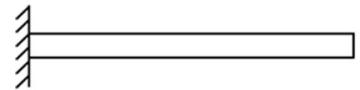

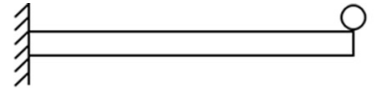

b

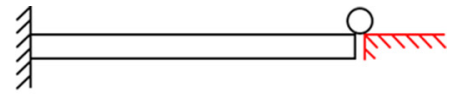

C

FIG. 1. Model of a plain cantilever (a), a cantilever with a particle on its free end (b), and a cantilever with a particle across its free end and a supporting structure (called a particle anchor) that constrains the motion of the particle (c).

and the effective mass of the cantilever is

$$
m_{\mathrm{eff} 1}=\frac{\rho w t L}{\lambda_{1}{ }^{4}},
$$

where $w$ is the width of the cantilever. If a particle is deposited onto the free end of the cantilever [see Fig. 1(b)], it will add an effective stiffness $k_{\text {eff }}$ and an effective mass $m_{\text {eff }}$, which can be expressed, respectively, as the relative effective stiffness added

$$
r_{k}=\frac{k_{\mathrm{eff} p}}{k_{\mathrm{eff} 1}}
$$

and the relative effective mass added

$$
r_{m}=\frac{m_{\mathrm{eff} p}}{m_{\mathrm{eff} 1}} .
$$

The resonant frequency of the cantilever with the particle added on the free end is then given by

$$
f_{p}=\frac{1}{2 \pi} \sqrt{\frac{k_{\mathrm{eff}_{1}}\left(1+r_{k}\right)}{m_{\mathrm{eff}_{1}}\left(1+r_{m}\right)}}
$$

and the resonant frequency shift is

$$
f_{p}-f_{0}=f_{0}\left(\sqrt{\frac{1+r_{k}}{1+r_{m}}}-1\right) .
$$

This equation shows that the frequency shift depends on $f_{0}$, $r_{k}$, and $r_{m}$. For conventional cantilevers, $r_{k}$ is usually negligible compared to $r_{m}$, since the mass-change effect of deposited particles often dominates the stiffness-change effect. ${ }^{23}$ Also, $r_{m}$ depends on the location of the particle; it increases as the particle approaches the free end of the cantilever. However, for cantilevers with a gap [see Fig. 1(c)], one can reasonably imagine $r_{k}$ dominating over $r_{m}$ when a particle is deposited across the gap, provided the stiffness of the bridge formed by the particle is important relative to the stiffness of the cantilever. Here, we assume that the boundary condition of the cantilever remains the same. In the case where $r_{k}$ dominates over $r_{m}$, we would expect the resonant frequency of cantilevers based on the gap method to increase rather than decrease. By comparing the resonant frequency shifts of cantilevers with a gap with conventional cantilevers, we can determine whether this stiffness-change approach is more sensitive than the conventional masschange approach. Since we are mainly interested in comparing cantilevers with a gap with conventional cantilevers, our analysis here ignores damping, and our subsequent experiments are performed in air, a medium with insignificant damping.

\section{Design}

We fabricated cantilevers on silicon-on-insulator chips using the PiezoMUMPs micromachining process $^{24}$ (see Fig. 2). The cantilevers are $200 \mu \mathrm{m}$ long and have a mass of $199 \mathrm{ng}$. The cantilevers have a $3 \mu \mathrm{m}$ gap between their free end and the particle anchor. This gap size is the smallest recommended size in the process handbook. The particle anchor is a large fixed-fixed beam. The cantilevers have a piezoelectric strip (aluminum nitride sandwiched between gold and doped silicon) at their fixed end and they are suspended above a trench. Applying voltage across the piezoelectric strip (across the gold and the doped silicon on the cantilever) causes the cantilevers to vibrate out of plane.

\section{Particle deposition}

Polystyrene microspheres were used for testing instead of bacteria since they are available in a variety of sizes and can be easily deposited onto and removed from silicon surfaces. We chose particles with a diameter of $6 \mu \mathrm{m}$, which is slightly larger than the gap size of the cantilever. The particles have an average mass of $\approx 120 \mathrm{pg} /$ particle (Polysciences Inc, 07312-5). We deposited the particles onto the surface of the cantilevers by placing a $10 \mu \mathrm{l}$ droplet of a solution of polystyrene microspheres, diluted with distilled water to a concentration of $10^{5}$ particles $/ \mathrm{ml}$, on the surface of the chip and letting the solution evaporate. Evaporation created favorable flow patterns for particle deposition both on the surface of the cantilevers and across the gap. In previous work, we have also showed that particles can be actively collected in the gap using electrokinetics instead of evaporation. ${ }^{25}$ We removed the particles from the surface of the cantilevers by slanting the chip and rinsing it vigorously with distilled water for $15 \mathrm{~s}$.

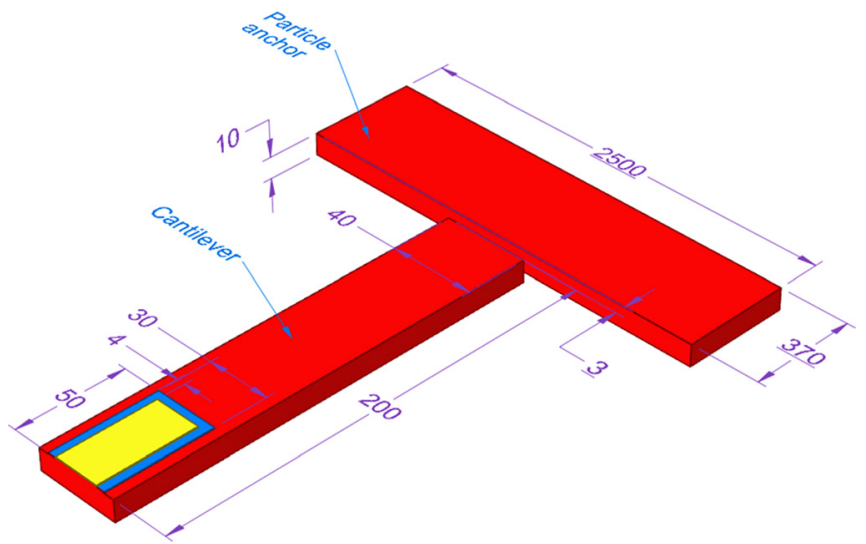

FIG. 2. Isometric drawing of the cantilever with a gap. The cantilever, the particle anchor, and the anchored surfaces are identified. The dimensions are in $\mu \mathrm{m}$. The underlined dimensions are not to scale. The material layers are color-coded: red is for silicon (doped at surface), blue is for aluminum nitride (piezoelectric material), and yellow is for gold. 


\section{RESULTS}

\section{Comparison between a conventional cantilever and a cantilever with a gap}

We first compared the change in the frequency response of a conventional cantilever with that of a cantilever with a gap in response to particle deposition. In the experiment, we measured the frequency response of both the conventional cantilever and the cantilever with a gap before depositing particles, after depositing them, and after rinsing them off. Both cantilevers have the same geometry, except that the conventional cantilever does not have a particle anchor near its free end, and it has a thin gold electrode that extends along its length in the center-this electrode has a negligible effect on the frequency response of the cantilever and was intended for another experiment. The cantilevers were excited with a periodic chirp signal with an amplitude of $3 \mathrm{~V}$, and the frequency response of a point on the free end of the cantilevers was measured from 50 to $400 \mathrm{kHz}$ with a vibrometer (Polytech MSA-400 Micro System Analyzer).

Figure 3 shows the zoomed-in frequency responses $(270-310 \mathrm{kHz})$ of the conventional cantilever and the cantilever with a gap and the corresponding images. Before depositing particles ( $b$ and e), each cantilever has a clean surface. The conventional cantilever has a resonant frequency of $294.9 \mathrm{kHz}$ and the cantilever with a gap has a resonant frequency of $289.6 \mathrm{kHz}$, and the amplitude at resonance for both cantilevers is $\approx 10^{-8} \mathrm{~m}$. Maps of out-of-plane displacements obtained with the vibrometer confirmed that these resonant frequencies represent the first bending mode of the cantilevers (see Refs. 26 and 27 for similar work). We consistently observed a slightly lower resonant frequency for the cantilever with a gap, which suggests that the thin air gap at the tip of the cantilever increases damping. After depositing particles (c and f), each cantilever has 8 particles scattered on its surface, and the cantilever with a gap has an additional particle across the gap. A zoomed-in image of the gap shows that this particle forms a bridge across the particle anchor and the left corner of the free end of the cantilever. As expected, the resonant frequency of the conventional cantilever drops by $0.9 \mathrm{kHz}$, since its effective mass of the cantilever has increased. In comparison, the resonant frequency of the cantilever with a gap is no longer observed, and the amplitude has dropped to $\approx 10^{-11} \mathrm{~m}$ over the frequency range shown. The resonant frequency of the cantilever with a gap did not appear either in the zoomed-out (50 to $400 \mathrm{kHz}$ ) frequency response (not shown), and the amplitude here remained well below $\approx 10^{-10} \mathrm{~m}$. This suggests that the deposition of a single particle across the gap increases the effective stiffness of the cantilever so significantly that it increases the resonant frequency of the cantilever above the measured range. It also suggests that the increase in the effective mass from the deposition of 8 other particles is insignificant compared to the increase in effective stiffness from the deposition of the single particle across the gap. Finally, after rinsing the particles off ( $d$ and $g$ ), all the particles are removed from the cantilevers, and the frequency response of each cantilever returns to its original state.

To see if the gap method produces a repeatable change in the frequency response, we performed the experiment several times and calculated the amplitude ratio obtained with each cantilever. The amplitude ratio, although based on amplitude shift, is related to the resonant frequency shift, and it can be used to measure the changes in frequency response. ${ }^{26,28}$ It is defined as $A_{i}\left(f_{i}\right) / A_{f}\left(f_{i}\right)$, where $A_{i}$ is the initial (clean cantilever) amplitude, $f_{i}$ is the initial (clean cantilever) resonant frequency, and $A_{f}$ is the final (cantilever with particles) amplitude. The amplitude ratio represents the change in amplitude at a fixed frequency. For example, in Fig. 3(a), for the conventional cantilever, $f_{i}=294.9 \mathrm{kHz}, \quad A_{i}\left(f_{i}\right)=1.225 \times 10^{-8} \mathrm{~m}, \quad$ and $A_{f}\left(f_{i}\right)=1.645$ $\times 10^{-9} \mathrm{~m}$, which gives an amplitude ratio of 7.5.

Figure 4 shows the amplitude ratios obtained for both cantilevers. When the conventional cantilever has 3 particles on it, it has an amplitude ratio of 4.5. In comparison, when the cantilever with a gap has 3 particles on it, it has an amplitude ratio of 1677.8 , which is more than two orders of magnitude higher than the corresponding value for the conventional cantilever. Overall, in all the tests, the amplitude ratio of the cantilever with a gap is one to three orders of magnitude higher than that of the conventional cantilever.
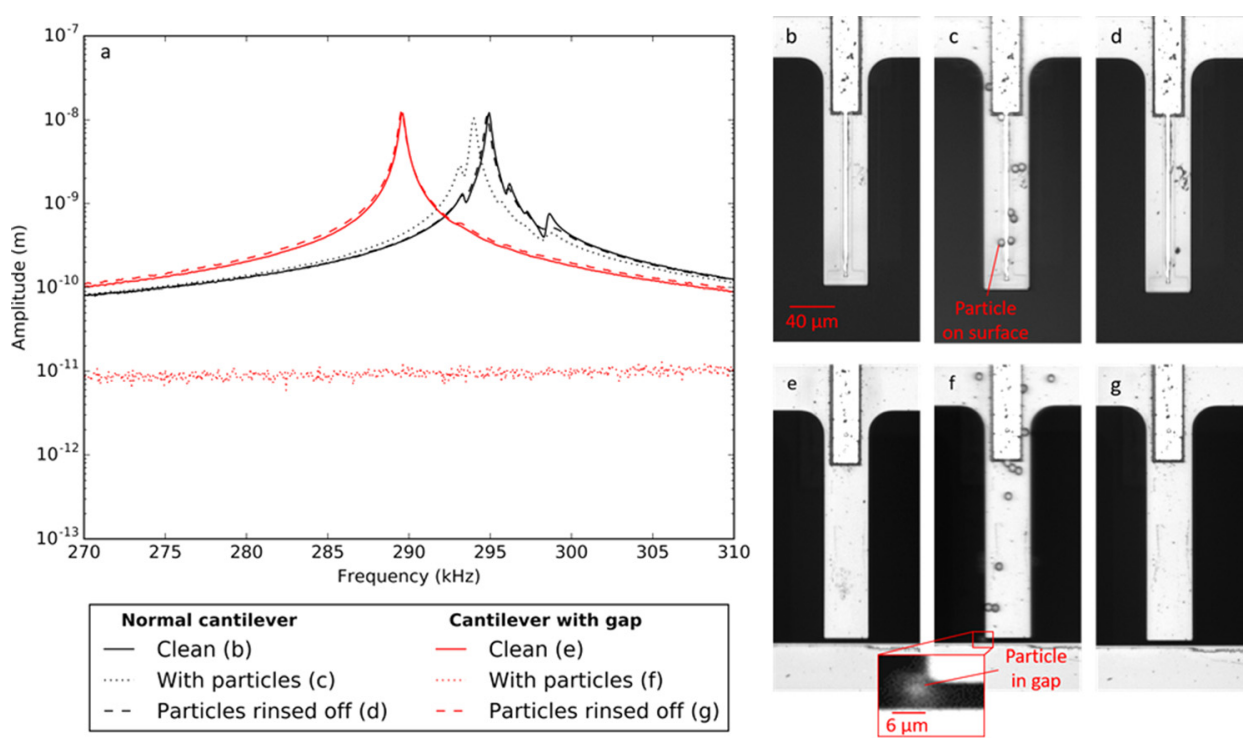

FIG. 3. (a) Zoomed-in frequency responses of a conventional cantilever and a cantilever with a gap when the cantilevers are clean, with particles, and when the particles are rinsed off. (b)-(g) Images that correspond to the frequency responses. 


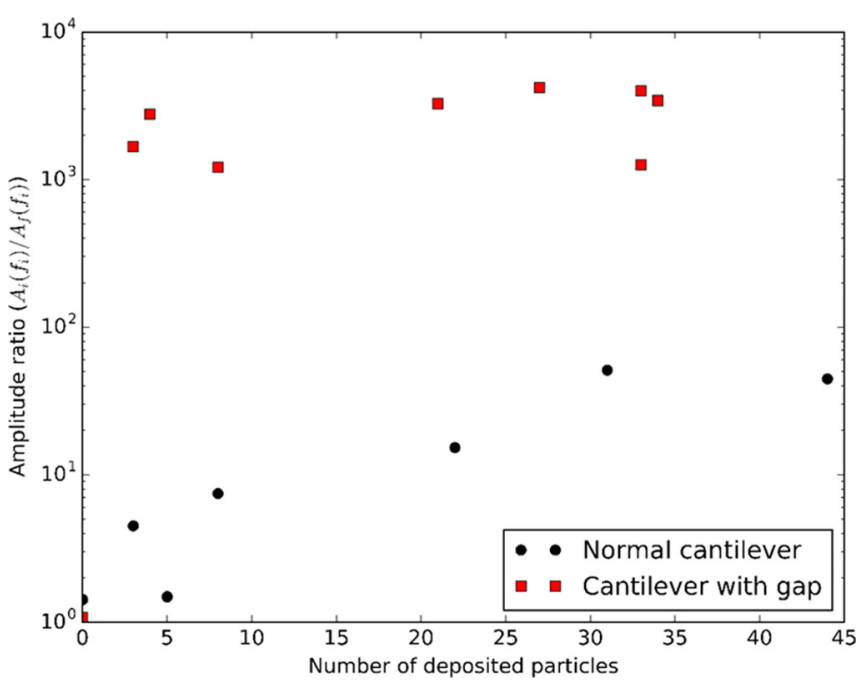

FIG. 4. Amplitude ratio of a conventional cantilever and a cantilever with a gap as a function of the number of particles deposited onto the cantilevers. The cantilever with a gap had between 1 and 5 particles in the gap in all the tests.

The amplitude ratio of the conventional cantilever increases as more particles are deposited onto its surface. We expect this, as more particles are deposited onto its surface, the effective mass of the cantilever increases. We note some variability in the trend, which we attribute to variations in the spatial distribution of the particles. When the spatial distribution is biased toward the free end of the cantilever, larger amplitude ratios are expected, and when it is biased toward the fixed end, smaller amplitude ratios are expected.

The amplitude ratio of the cantilever with a gap jumps to a much higher value than the conventional cantilever when the number of deposited particles goes from 0 to 3 . As the number of deposited particles further increases, the slope of the cantilever with a gap levels off, especially in comparison to the slope of the conventional cantilever. We think the main reason for this being that the deposition of a single particle across the gap causes the amplitude ratio of the cantilever with a gap to start to saturate. It is established in the literature that cantilever biosensors become less sensitive as the added mass increases. ${ }^{29}$ Similarly, we think that the cantilever with a gap becomes less sensitive as the added stiffness from the particles deposited across the gap increases. We also note that in any given test, although there are anywhere between 3 and 34 particles on the surface of the cantilever with a gap, due to the random spatial distribution of the particles, there are only between 1 and 5 particles across the gap itself. Since the change in the frequency response of the cantilever with a gap seems to be more closely related to the number of particles deposited across the gap rather than the total number of particles on the surface of the cantilever, we investigate the amplitude ratio as a function of the number of particles across the gap. Here, as expected, we observed a stronger trend and a sharper slope between the number of deposited particles and the amplitude ratio.

\section{Average frequency response}

In the previous experiments, the frequency response of a single point on the free end of the cantilever with a gap was measured from 50 to $400 \mathrm{kHz}$. To better understand how the frequency response of the cantilever with a gap was being changed by the deposition of particles across the gap, we performed a second experiment. We measured the average frequency response of the entire surface of the cantilever instead of only measuring a single point, and we increased the measured frequency range from $50-400 \mathrm{kHz}$ and from $50-2000 \mathrm{kHz}$.

Figure 5 shows the average frequency response of the cantilever and the corresponding image of the gap for five tests. In the first test (b), there are no particles across the gap and the frequency response of the cantilever is typical. The peak at $289.1 \mathrm{kHz}$ is the first bending mode and the peak at $1264.0 \mathrm{kHz}$ is the second bending mode. Maps of out-ofplane displacements obtained with the vibrometer confirmed the mode shapes.

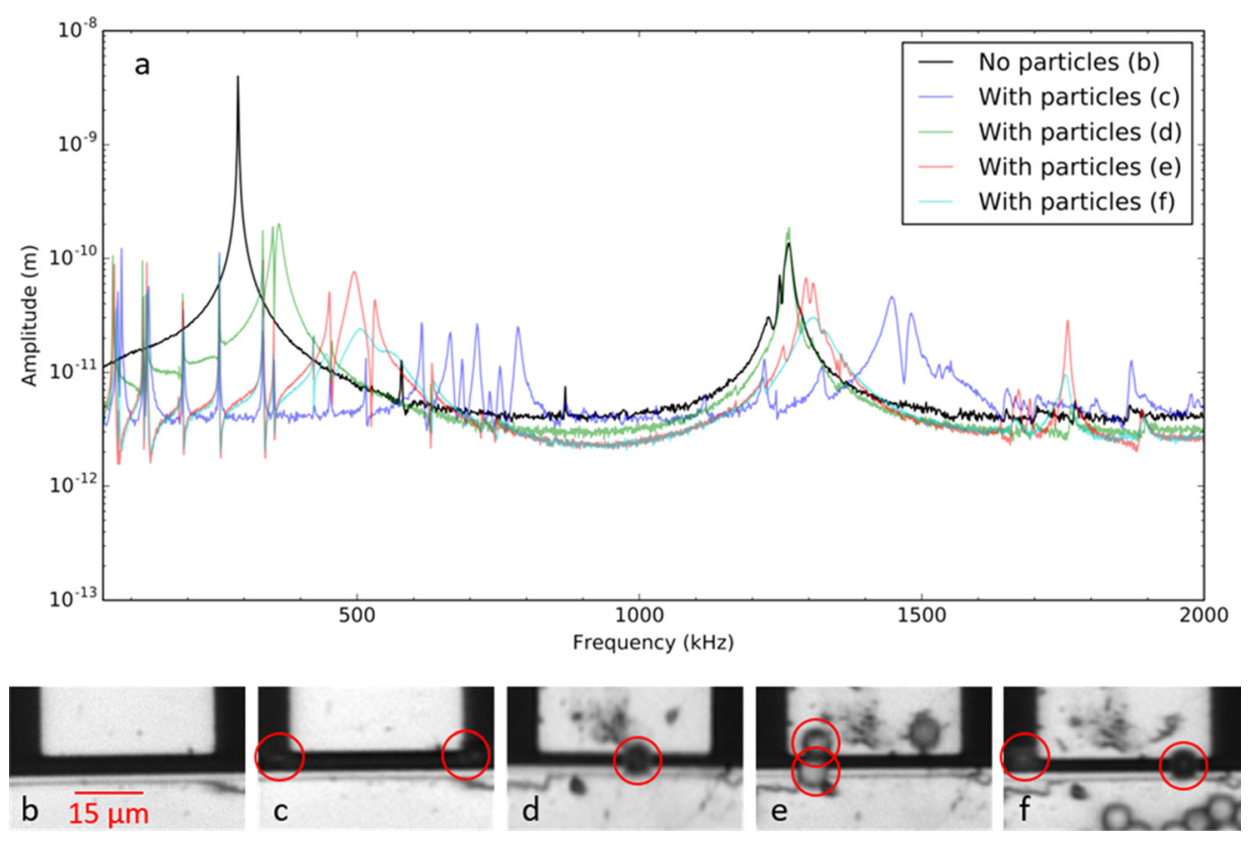

FIG. 5. (a) Average frequency response of a cantilever with a gap when the cantilever has no particles on it and when it has particles across the gap. (b)-(f) Close-up images of the gap that correspond to the frequency responses. The particles are encircled. 
In the next four tests (c to f), there is either one or two particles across the gap. Instead of obtaining a slight decrease in both resonant frequencies, as we would expect with a conventional cantilever, we obtain a host of new low frequency peaks around the original peak of the first bending mode (between 0 and $500 \mathrm{kHz}$ ) and a single dominant peak above each of the original peaks of the first and second bending modes. The low frequency peaks are the result of structural coupling between the cantilever and the particle anchor through the particle bound across the gap. The particle anchor is a large fixed-fixed beam with many resonant frequencies in this frequency range.

The dominant peaks above each of the original peaks of the first and second bending modes, with their broad shape and high amplitude, closely resemble the original peaks (especially d, e, and f) and they do not overlap each other between tests. Thus, these peaks can be identified as the first and second bending modes of the cantilever. Using spectrum (e) as a reference, we can make the following observations. The resonant frequency of the first bending mode has increased by $205.6 \mathrm{kHz}$, which is over two orders of magnitude higher than the $0.9 \mathrm{kHz}$ increase we obtained previously with the conventional cantilever (see Fig. 3). The resonant frequency of the second bending mode has increased by $30.9 \mathrm{kHz}$, which is much lower than the first bending mode. This suggests that the effective stiffness added by the particles is the highest for the first bending mode.

\section{Simulation}

To validate our analysis of the gap method, we simulated, using COMSOL Multiphysics, the average frequency response of the cantilever under different loading conditions. We used the same geometry and materials for the cantilever and the particle anchor as we had in our experiments. We modeled the polystyrene particle as a cube ( $6 \mu$ m side length) rigidly attached to surfaces. To excite the cantilever, we applied an electric potential of $3 \mathrm{~V}$ across the piezoelectric strip from 1 to $2 \mathrm{MHz}$ with a step size of $2 \mathrm{kHz}$. We used a step size of $0.05 \mathrm{kHz}$ near the first resonant frequency of the cantilever to account for the sharpness of the peak. We simulated damping with the Rayleigh damping model using the experimental resonant frequencies and quality factors of the first two bending modes of the cantilever as input. We also performed a simulation of the particle anchor without the cantilever to verify that the lower resonant frequencies we observed in Fig. 3 are associated with the particle anchor. To excite the particle anchor, we applied a prescribed positive vertical displacement of $1 \mathrm{~nm}$ on the top surface of it. Figure 6 shows the results of our simulation. The average frequency response and the fixed displacement boundary conditions are shown for five different loading conditions.

With no particle on the cantilever (b), the resonant frequency of the first bending mode is $287.7 \mathrm{kHz}$, and the resonant frequency of the second bending mode is $1538.0 \mathrm{kHz}$. The error $\delta=\left|1-f_{s} / f\right|$, where $f_{s}$ is the simulated resonant frequency and $f$ is the experimental resonant frequency, is $0.5 \%$ for the first bending mode and $21.7 \%$ for the second bending mode. The simulated peak amplitude $\left(\approx 10^{-6} \mathrm{~m}\right)$ is higher than the experimental peak amplitude $\left(\approx 10^{-8} \mathrm{~m}\right)$. We expected this, since in the simulation, the sinusoidal excitation signal concentrates the energy at a fixed frequency, while in our experiments, the periodic chirp excitation signal distributes the energy over a wide frequency range.

With a particle on the free end of the cantilever (c), the resonant frequencies decrease by $0.7 \mathrm{kHz}$ and $6.0 \mathrm{kHz}$ respectively. The drop in the first resonant frequency is close to the $0.9 \mathrm{kHz}$ drop we obtained experimentally (see Fig. 3).

With a particle in the gap with our actual design (d), the resonant frequencies of the particle anchor appear, and the resonant frequencies of the cantilever increase by $353.0 \mathrm{kHz}$ and $144.0 \mathrm{kHz}$, respectively. The resonant frequency shifts

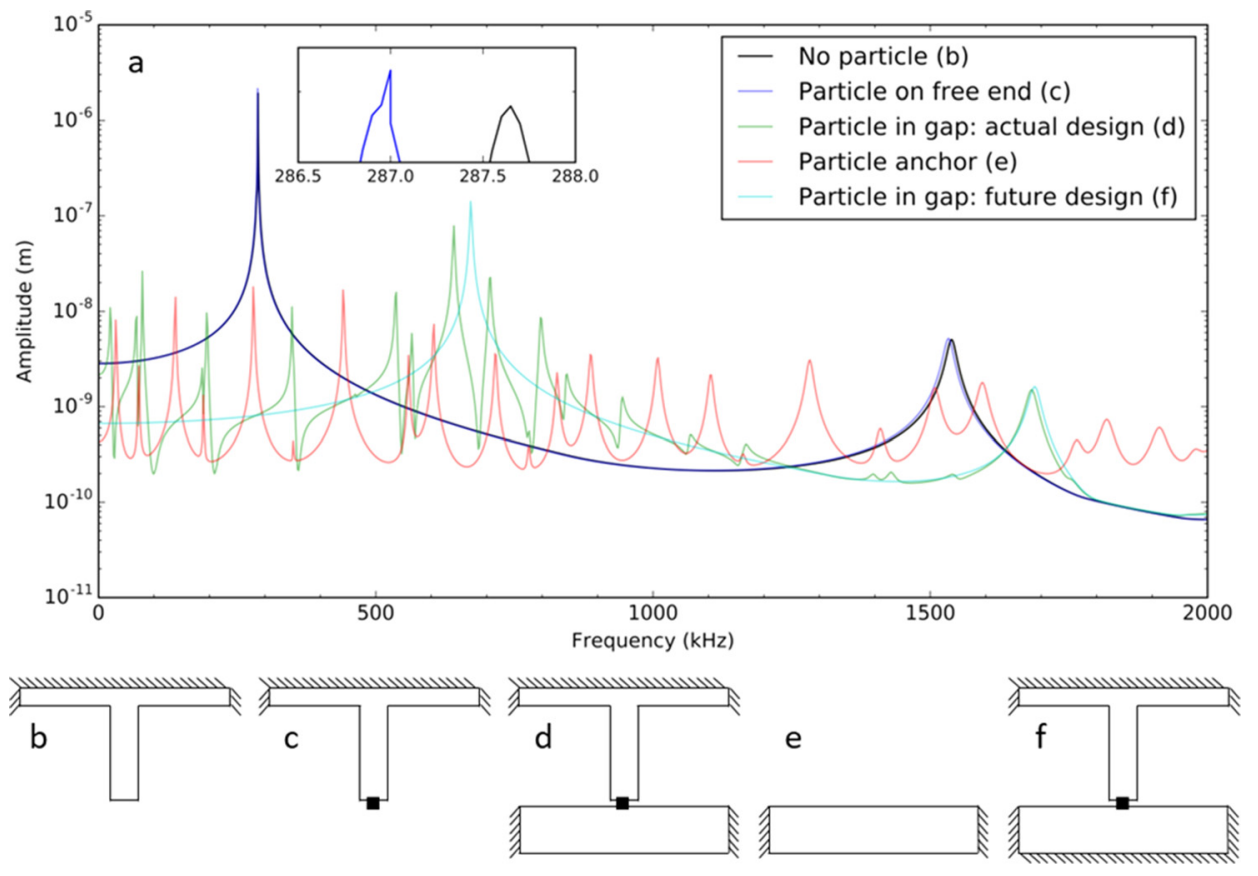

FIG. 6. (a) Simulated average frequency response of the cantilever with a gap or the particle anchor under different loading conditions. A zoomed-in inset of the first resonant frequency of the cantilever is included. (b)-(f) Schematic of the models that correspond to the average frequency responses. 
obtained in the simulation are higher than those obtained in our experiments (see Fig. 3). We expect this since in the simulation the particle is rigidly connected to surfaces, while in the experiments, the adhesion forces between the particle and the surfaces are certainly weaker than a rigid connection.

In the simulation of the particle anchor without the cantilever (e), we see that many lower resonant frequencies appear, and they roughly align with those seen in (d). This supports our conclusion that the lower resonant frequencies observed in Fig. 3 are associated with the particle anchor and not the cantilever.

Finally, in the simulation of a future design in which the particle anchor is anchored along its length at one end and there is a particle across the gap (f), the lower resonant frequencies of the particle anchor disappear and the resonant frequencies of the cantilever increase by $384.0 \mathrm{kHz}$ and $150.0 \mathrm{kHz}$, respectively. By anchoring the particle anchor along its length at one end, we increase the resonant frequencies of the particle anchor beyond the frequency range in the simulation. This eliminates interference between the resonant frequencies of the particle anchor and those of the cantilever. We also increase the shift in the resonant frequencies of the cantilever, since less energy can be dissipated through the particle anchor when it is anchored.

\section{CONCLUSION}

In conclusion, we have presented a gap method that can greatly increase the sensitivity of dynamic-mode cantilever biosensors. This method seems to rely on the change in effective stiffness of the cantilever instead of the change in its effective mass. When a single polystyrene particle with a diameter of $6 \mu \mathrm{m}$ forms a bridge across the free end of the cantilever and the particle anchor, it generates a great change in the frequency response of the cantilever. By analyzing the average frequency response of the cantilever, we determined that the particle in the gap greatly increases the resonant frequency of the first bending mode of the cantilever and induces structural coupling between the cantilever and the particle anchor. Simulations showed that the resonant frequencies of the particle anchor, which interfere with the frequency response of the cantilever, can be eliminated by anchoring the particle anchor along its length at one end. The gap method can be easily integrated into micromachined cantilever designs; it simply requires a particle anchor to be placed $3 \mu \mathrm{m}$ away from the free end of the cantilever. The current limitation of the gap method is that it would not work well for applications requiring the measurement of particles smaller than the gap size. However, there are many applications requiring the measurement of particles larger than the gap size, such as large bacteria and eukaryotic cells. ${ }^{30}$ Future work will involve developing an anchored particle anchor design and studying the gap method in water.

\section{ACKNOWLEDGMENTS}

The authors acknowledge NSERC for funding and CMC Microsystems for fabrication support.
${ }^{1}$ N. V. Lavrik, M. J. Sepaniak, and P. G. Datskos, "Cantilever transducers as a platform for chemical and biological sensors," Rev. Sci. Instrum. 75(7), 2229 (2004).

${ }^{2}$ C. Ziegler, "Cantilever-based biosensors," Anal. Bioanal. Chem. 379(7-8), 946-959 (2004).

${ }^{3}$ M. Alvarez and L. M. Lechuga, "Microcantilever-based platforms as biosensing tools," 827-836 (2010).

${ }^{4}$ J. L. Arlett, E. B. Myers, and M. L. Roukes, "Comparative advantages of mechanical biosensors," Nat. Nanotechnol. 6(4), 203-215 (2011).

${ }^{5}$ W. Pang, H. Zhao, E. S. Kim, H. Zhang, H. Yu, and X. Hu, "Piezoelectric microelectromechanical resonant sensors for chemical and biological detection," Lab Chip 12(1), 29 (2012).

${ }^{6} \mathrm{R}$. Lalauze, Chemical Sensors and Biosensors (ISTE Ltd., London, 2012). ${ }^{7}$ B. Ilic, D. Czaplewski, M. Zalalutdinov, H. G. Craighead, P. Neuzil, C. Campagnolo, and C. Batt, "Single cell detection with micromechanical oscillators," J. Vac. Sci. Technol. B Microelectron. Nanometer Struct. 19(6), 2825 (2001).

${ }^{8}$ A. Gupta, D. Akin, and R. Bashir, "Single virus particle mass detection using microresonators with nanoscale thickness," Appl. Phys. Lett. 84(11), 1976 (2004).

${ }^{9}$ B. Ilic, "Attogram detection using nanoelectromechanical oscillators," J. Appl. Phys. 95(7), 3694 (2004).

${ }^{10}$ Y. T. Yang, C. Callegari, X. L. Feng, K. L. Ekinci, and M. L. Roukes, "Zeptogram-scale nanomechanical mass sensing," Nano Lett. 6(4), 583-586 (2006).

${ }^{11}$ J. E. Sader, "Frequency response of cantilever beams immersed in viscous fluids with applications to the atomic force microscope," J. Appl. Phys. 84(1), 64 (1998).

${ }^{12}$ Y. Liu, X. Li, Z. Zhang, G. Zuo, Z. Cheng, and H. Yu, "Nanogram per milliliter-level immunologic detection of alpha-fetoprotein with integrated rotating-resonance microcantilevers for early-stage diagnosis of heptocellular carcinoma," Biomed. Microdevices 11(1), 183-191 (2009).

${ }^{13}$ Y. Tao, X. Li, T. Xu, H. Yu, P. Xu, B. Xiong, and C. Wei, "Resonant cantilever sensors operated in a high-Q in-plane mode for real-time bio/chemical detection in liquids," Sens. Actuators, B 157(2), 606-614 (2011).

${ }^{14}$ D. Maraldo, K. Rijal, G. Campbell, and R. Mutharasan, "Method for labelfree detection of femtogram quantities of biologics in flowing liquid samples," Anal. Chem. 79(7), 2762-2770 (2007).

${ }^{15} \mathrm{G}$. A. Campbell and R. Mutharasan, "Detection of pathogen Escherichia coli O157:H7 using self-excited PZT-glass microcantilevers," Biosens. Bioelectron. 21(3), 462-473 (2005).

${ }^{16}$ C. Ricciardi, G. Canavese, R. Castagna, I. Ferrante, A. Ricci, S. L. Marasso, L. Napione, and F. Bussolino, "Integration of microfluidic and cantilever technology for biosensing application in liquid environment," Biosens. Bioelectron. 26(4), 1565-1570 (2010).

${ }^{17}$ B. N. Johnson and R. Mutharasan, "A cantilever biosensor-based assay for toxin-producing cyanobacteria microcystis aeruginosa using 16S rRNA," Environ. Sci. Technol. 47, 12333-12341 (2013).

${ }^{18} \mathrm{H}$. Sharma and R. Mutharasan, "Review of biosensors for foodborne pathogens and toxins," Sens. Actuators, B 183, 535-549 (2013).

${ }^{19}$ B. N. Johnson and R. Mutharasan, "Sample preparation-free, real-time detection of microRNA in human serum using piezoelectric cantilever biosensors at attomole level," Anal. Chem. 84(23), 10426-10436 (2012).

${ }^{20} \mathrm{~S}$. Xu and R. Mutharasan, "Rapid and sensitive detection of giardia Lamblia using a piezoelectric cantilever biosensor in finished and source waters," Environ. Sci. Technol. 44(5), 1736-1741 (2010).

${ }^{21}$ R. A. Barton, B. Ilic, S. S. Verbridge, B. R. Cipriany, J. M. Parpia, and H. G. Craighead, "Fabrication of a nanomechanical mass sensor containing a nanofluidic channel," Nano Lett. 10(6), 2058-2063 (2010).

${ }^{22}$ T. P. Burg, M. Godin, S. M. Knudsen, W. Shen, G. Carlson, J. S. Foster, K. Babcock, and S. R. Manalis, "Weighing of biomolecules, single cells and single nanoparticles in fluid," Nature 446(7139), 1066-1069 (2007).

${ }^{23} \mathrm{~B}$. N. Johnson and R. Mutharasan, "Biosensing using dynamic-mode cantilever sensors: A review," Biosens. Bioelectron. 32(1), 1-18 (2012).

${ }^{24}$ A. Cowen, G. Hames, K. Glukh, and B. Hardy, PiezoMUMPs Design Handbook (MEMSCAP Inc., 2013).

${ }^{25}$ S. Leahy and Y. Lai, "A cantilever biosensor based on a gap method for detecting Escherichia coli in real time," Sens. Actuators, B 246, 1011-1016 (2017).

${ }^{26}$ S. Leahy and Y. Lai, "An hourglass design with electrokinetic sampling and electrothermal actuation for micro biosensors," Sens. Actuators, B 223, 123-130 (2016). 
${ }^{27}$ S. Leahy and Y. Lai, "A cantilever biosensor exploiting electrokinetic capture to detect Escherichia coli in real time," Sens. Actuators, B 238, 292-297 (2017).

${ }^{28}$ R. S. Lakshmanan, S. Xu, and R. Mutharasan, "Impedance change as an alternate measure of resonant frequency shift of piezoelectric-excited millimetersized cantilever (PEMC) sensors," Sens. Actuators, B 145(1), 601-604 (2010).
${ }^{29}$ D. Maraldo and R. Mutharasan, "Mass-change sensitivity of piezoelectricexcited millimeter-sized cantilever (PEMC) sensors: Model and experiments," Sens. Actuators, B 132(1), 140-148 (2008).

${ }^{30}$ A. Ahmed, J. V. Rushworth, N. A. Hirst, and P. A. Millner, "Biosensors for whole-cell bacterial detection," Clin. Microbiol. Rev. 27(3), 631-646 (2014). 